\title{
Experimental Study of Improved Watermarking Scheme on Relational Databases
}

\author{
N.G. Pardeshi \\ Assistant Professor \\ University of Pune \\ Department of Computer \\ Engineering \\ SRESCOE Kopargaon-423603
}

\author{
J.N. Kale \\ Assistant Professor \\ University of Pune \\ Department of Computer \\ Engineering \\ SRESCOE Kopargaon-423603
}

\author{
M.S. Ankoshe \\ Assistant Professor \\ University of Pune \\ Department of Computer \\ Engineering \\ SRESCOE Kopargaon-423603
}

\begin{abstract}
The main objective of watermarking is to protect a digital content from unauthorized redistribution and copying by enabling ownership provability over the content. The goal of digital watermarking is to insert a robust and imperceptible watermark into the digital content such that the mark does not destroy the value of the content, and the mark is hard to be removed by attackers without destroying the utility of content. The measurement of the value of the content is closely related to the data type and its intended use.
\end{abstract}

We have proposed a new marking scheme called as improved watermarking. Watermarking scheme first generates the bit string of fixed length. The mark bit is computed with the help of watermark bit and mask bit, which is used to mark the certain bit location of the attribute value.

\section{General Terms}

Relational Database Security, Improved Watermarking on Relational Database

\section{Keywords}

Digital watermarking, relational database systems, copyright protection, ownership verification, imperceptibility, robustness, Watermark embedding, Watermark extraction.

\section{INTRODUCTION}

The watermarking software introduces small errors into the object being watermarked. These intentional errors are called marks and all the marks together compose the watermark. The marks must not have a significant impact on the usefulness of the data and they should be placed in such a way that a malicious user cannot destroy them without making the data less useful. Thus, watermarking does not prevent copying, but it prevents illegal copying by providing a means for establishing the original ownership of a redistributed copy [1] [2] [3]. Most of these techniques were initially developed for still images [4] and then extended to video [5] and audio sources [6] [7].

The increasing use of databases in applications is creating a similar need for watermarking databases. For example, in the semiconductor industry, parametric data on semiconductor parts is provided primarily by three companies: Aspect, IHS, and IC Master. They all pay a large number of people to manually extract part specifications from datasheets. They then license these databases at high prices to design engineers. Companies like Acxiom have compiled large collections of consumer and business data. In the life sciences industry, the primary assets of companies such as Celera are the databases of biological information. The Internet is exerting huge pressure on these data providers to create services that allow users to search and access databases remotely. While this trend is a fortunate thing to end users, it is exposing the data providers to the threat of data theft. They are therefore demanding capabilities for identifying pirated copies of their data. Watermarking on text data [8] and software [9] differs from database watermarking.

We suggest that rights management of relational data through watermarking should become an important topic for database research. Database relations that can be watermarked have attributes which are such that changes in a few values do not affect the applications. But are there real-world datasets that can tolerate a small amount of error without degrading their usability? Consider the ACARS meteorological data used in building weather prediction models [10]. The wind vector and temperature accuracies in this data are estimated to be within $1.8 \mathrm{~m} / \mathrm{s}$ and $0.5^{0} \mathrm{C}$ respectively [10]. The errors introduced by watermarking can easily be constrained to lie within the measurement tolerance in this data. Later in the paper, we report experimental results using a forest cover dataset. It contains measurements for variables such as elevation, aspect, slope, distance to hydrology and roadways, soil type, etc. Small changes in some of the measurements do not affect the usability of this data.

\section{WATERMARKING MODEL}

In general, any watermarking system consists of three modules such as watermark embedding, attacker channel, and watermark extraction. Each module along with its input and output is shown in the figure 1 .

\subsection{Watermark Embedding}

Embedding module is responsible for insertion of watermark bit string into the relational database. Generally inputs for embedding module are i) watermark bit string to be embedded, ii) original database iii) secret key. By using the insertion algorithm, certain bit locations in the database are altered. Output of encoder module is watermarked database.

\subsection{Attacker Channel}

As watermarked database is publically available for use, it can be accessed by number of database users. If certain users are malicious, they can try to destroy the original watermark and insert their own. Thus watermarked database can undergo with different types of attacks such as subset selection, subset deletion, and subset alteration. 


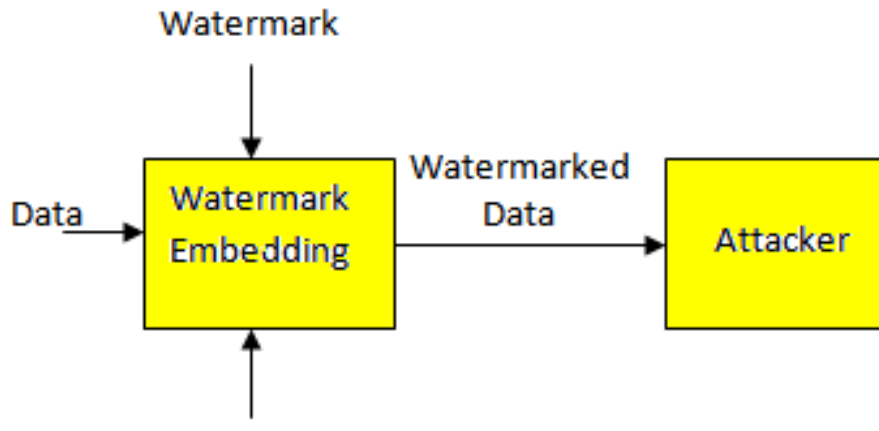

Secret Key

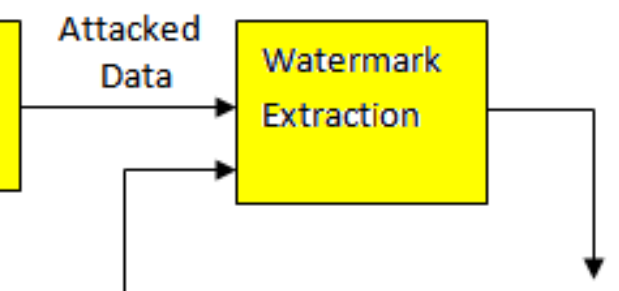

Extracted Watermark

Figure 1: Watermarking Model

\subsection{Watermark Extraction}

Input for extraction module is watermarked database. Extraction module is responsible for detecting the watermark bit string in the marked database. Detection algorithm verifies the bit locations which are marked by the insertion algorithm and validates the identity of the mark. Output of decoder module is extracted watermark bit string from the database.

\section{WATERMARKING SYSTEM}

Watermarking scheme consists of two algorithms, watermark insertion and watermark detection. Our improved watermarking scheme is developed by extending the watermarking scheme, which was proposed by Agrawal et al. for watermarking relational databases [11] [12].

Consider a database relation $R$ with primary key $P$ and $v$ numerical attributes $A_{0}, \ldots, A_{v-1}$. Assume that it is acceptable to change one of $\rho$ least significant bits in a small number of numeric values. The relation has $\eta$ tuples and a fraction $1 / \gamma$ of them will be used for watermarking.

The owner of $R$ has a secret key $K$. A cryptographic pseudorandom sequence generator [13] [14] $S$ is used to select tuples, attributes, bits, and decide how to change the bits in watermarking. Such a pseudorandom sequence generator produces a sequence of numbers from an initial seed. Without the knowledge of the seed, it is infeasible to compute the next number in the sequence. Different seeds lead to different sequences. For each tuple $r, S$ is seeded with the primary key $r . P$ concatenated with the secret key. Let $S_{i}(K, r . P)$ denote the $i^{\text {th }}$ number in the sequence generated by $S$. The values of $S_{i}(K$, $r . P)$ are uniformly distributed for different primary key values.

\subsection{Watermark Insertion}

The algorithm as shown in figure 2 inserts the watermark of buyer $n$ into relation $R$. For each tuple $r$ of $R$, the algorithm seeds the sequence generator $S$ with the concatenation of the secret key $K$ and the primary key r.P of the tuple. If the first sequence number $S_{I}(K, r . P) \bmod \gamma=0$, the tuple is selected. Therefore, on average, one out of $\gamma$ tuples are selected. For each selected tuple, the algorithm selects exactly one attribute; in particular, it selects attribute $i$ if $S_{2}(K, r . P) \bmod v=i$. Similarly, it selects least significant bit $j$ from the selected attribute if $S_{3}(K, r . P)$ mod $\rho=j$. On the other hand, the algorithm computes a mask bit $x$ according to $S_{4}(K, r . P)$; if $S_{4}(K, r . P)$ is even, then $x=0$ else $x=1$. It also selects a watermark bit $f_{l}$ if $S_{5}(K, r . P) \bmod L=l$. Finally, the algorithm XOR's the watermark bit with the mask bit and assigns the selected bit with the XOR'ing result. The purpose of using mask bits is to hide the distribution of watermark bits. Note that all selections and computations in watermark insertion are based on $S_{i}(K, r . P)$ which are uniformly distributed. On average, $\eta / \gamma$ bits (or tuples) are used to embed a watermark, and each watermark bit $f_{l}$ is embedded $\eta /(\gamma L)$ times. Also, note that the secret key is involved in every step of the process. Without knowing the secret key and without comparing multiple watermark copies, buyers are prevented from knowing where the watermark is embedded.

Input:

1. The relation $R$ with scheme $\left(P, A_{0}, \ldots, A_{v-1}\right)$

2. The private key $K$ is known only to the owner of the database.

3. The parameters $\gamma, v$, and $\rho$ are also private to the owner.

4. Watermark of buyer n: $F(K, n)=H(K \mid n)$

$$
/ / F(K, n)=\left(f_{0}, \ldots, f_{L-1}\right)
$$

Output:

1. Watermarked relation $R$

Algorithm:

1) for each tuple $r \in R$ do

2) if $\left(S_{I}(K, r . P) \bmod \gamma\right.$ equals 0$)$ then $/ /$ mark this tuple

3) attribute_index $i=S_{2}(K, r . P) \bmod v$ // mark attribute $A_{i}$

4) bit_index $j=S_{3}(K, r . P) \bmod \rho$

// mark least significant bit $j$

5) mask_bit $x=0$ if $S_{4}(K, r . P)$ is even; $x=1$ otherwise

6) watermark_index $l=S_{5}(K, r . P) \bmod L$

7) watermark_bit $f=f_{l}$

//select watermark bit $l$

8) mark_bit $\mathrm{m}=x \oplus f$

9) set least significant bit $j$ of $r \cdot A_{i}$ to $m$.

10) return $R$

Figure 2: Watermark Insertion Algorithm

\subsection{Watermark Detection}

In watermark detection, a merchant of database relation $R$ would like to determine whether another relation $R^{\prime}$ was pirated from $R$ and, if so, identify the attacker who distributed $R^{\prime}$ without authorization. If $R^{\prime}$ is pirated, the algorithm 
assumes that the primary key attribute values as well as the order among marked attributes have not changed (or else can be recovered). Note that $R$ ' may consist of only a subset of original tuples; it may include some additional tuples that were not in $R$, and some bit values in $R^{\prime}$ could have been changed by the attacker before detection.

The algorithm as in figure 3 initiates a watermark template $F$ $=\left(f_{0}, \ldots, f_{L-1}\right)$ as $(?, \ldots, ?)$, where "?" indicates that a mark bit is in an unreadable state. It then locates the marked bits exactly as the insertion algorithm does. From each marked bit, the algorithm extracts a watermark bit $f_{l}$ by XOR'ing the bit value with a computed mask bit. If the marked bit has been changed by the attacker, the extracted $f_{l}$ may not match its original value. The algorithm uses two counting variables count $[l][0]$ and count $[l][1]$ to indicate the number of times that $f_{l}$ is extracted to be 0 and 1 , respectively. After all marked bits are checked, the algorithm assigns 0 (or 1, respectively) to $f_{l}$ if the ratio count $[i][0] /(\operatorname{count}[i][0]+\operatorname{count}[i][1])$ (or $\operatorname{count}[i][1] /(\operatorname{count}[i][0]+\operatorname{count}[i][1])$, respectively) is greater than $\tau$, where $\tau \epsilon(0.5,1)$ is a real parameter that is related to the assurance of the detection process.

A attacker is detected in a subroutine detect if the recovered watermark template $F=\left(f_{0}, \ldots, f_{L-1}\right)$ matches one of the $N$ buyers' watermarks, which is computed in the same manner as the insertion algorithm.

\section{Input:}

1. The watermarked relation $R^{\prime}$ with scheme $\left(P, A_{0}, \ldots\right.$ ., $\left.A_{v-1}\right)$

2. $K, \gamma, v, \rho$ are the same as in watermark insertion

\section{Output:}

1. Detected watermark bit string

2. Buyer number

\section{Algorithm:}

1)// initiate watermark template and counts

2) watermark template $F=\left(f_{0}, \ldots, f_{L-1}\right)=(?, \ldots$, ? $)$

3) $\quad / / ? ' / /$ represent an unknown value

4) for each $i=0$ to $L-1$ do $\operatorname{count}[i][0]=\operatorname{count}[i][1]=0$

5) $\quad / / \operatorname{count}[i][0]$, count $[i][1]$ are votes for $f_{i}$ to be 0 and 1 respectively

6) // scan all tuples and obtain counts for each watermark bit

\section{7) for each tuple $r \in R^{\prime}$ do}

8) if $\left(S_{l}(K, r . P) \bmod \gamma\right.$ equals 0$)$ then

// this tuple was marked

9) attribute_index $i=S_{2}(K, r . P) \bmod v$

// attribute $A_{i}$ was marked

10) bit_index $j=S_{3}(K, r . P) \bmod \rho$ // bit $j$ was marked

11) if least significant bit $j$ of $r . A_{i}$ does not exist, then skip to the next tuple
14)

16) watermark_index $i=S_{5}(K, r . P) \bmod L$

17) $\operatorname{count}[i][f]=\operatorname{count}[i][f]+1$

// update the votes

18) // recover watermark

19) for each $i=0$ to $L-1$ do

20) if $\operatorname{count}[i][0]+\operatorname{count}[i][1]=0$ then return none suspected

21) $f_{i}=0$ if $\operatorname{count}[i][0] /(\operatorname{count}[i][0]+\operatorname{count}[i][1])>\tau$;

22) $f_{i}=1$ if $\operatorname{count}[i][1] /(\operatorname{count}[i][0]+\operatorname{count}[i][1])>\tau$;

23) return none suspected otherwise

24) $F=\left(f_{0}, \ldots, f_{L-1}\right)$

25) // determine a attacker

26) Buyer $n=\operatorname{detect}(F, K, L, N)$ attacker based on template $F$

// detect a

27) if $n \geq 0$ then return buyer $n$ is the attacker

28) else return none suspected

29) // subroutine: detect a attacker

30) detect (template $F$, secret key $K$, watermark length $L$, number of buyers $N$ )

31) for each buyer $n=0$ to $N-1$ do

32)

$F^{\prime}=H(K \mid n)$

33)

if $F$ matches $F^{\prime}$ then return $n$

34) return - 1

\section{Figure 3: Watermark Detection Algorithm}

\section{EXPERIMENTAL STUDY}

This section focuses on the results of proposed improved watermarking system for relational databases. Experiments are performed using the Forest Cover Type dataset, available from the University of California-Irvine KDD Archive.

http://www.kdd.ics.uci.edu/databases/covertype/covertype.ht $\mathrm{ml}$.

The dataset has 5,81,012 rows, each with 61 attributes. We have added an extra attribute called $i d$ to serve as the primary key. We have selected the first ten integer-valued attributes along with first $1,00,000$ tuples as candidates for watermarking. We assume that the primary key of the relation $R$ consists of a single attribute $P$ and 10 attributes are available for watermarking. Forest Cover Type dataset schema is as shown in figure 4 and detailed snapshot is as in figure 5 .

Watermark insertion as well as detection algorithms are implemented by using JAVA on Net Beans IDE 6.1RC2, along with database program MySQL server 5.1 running at back end. 


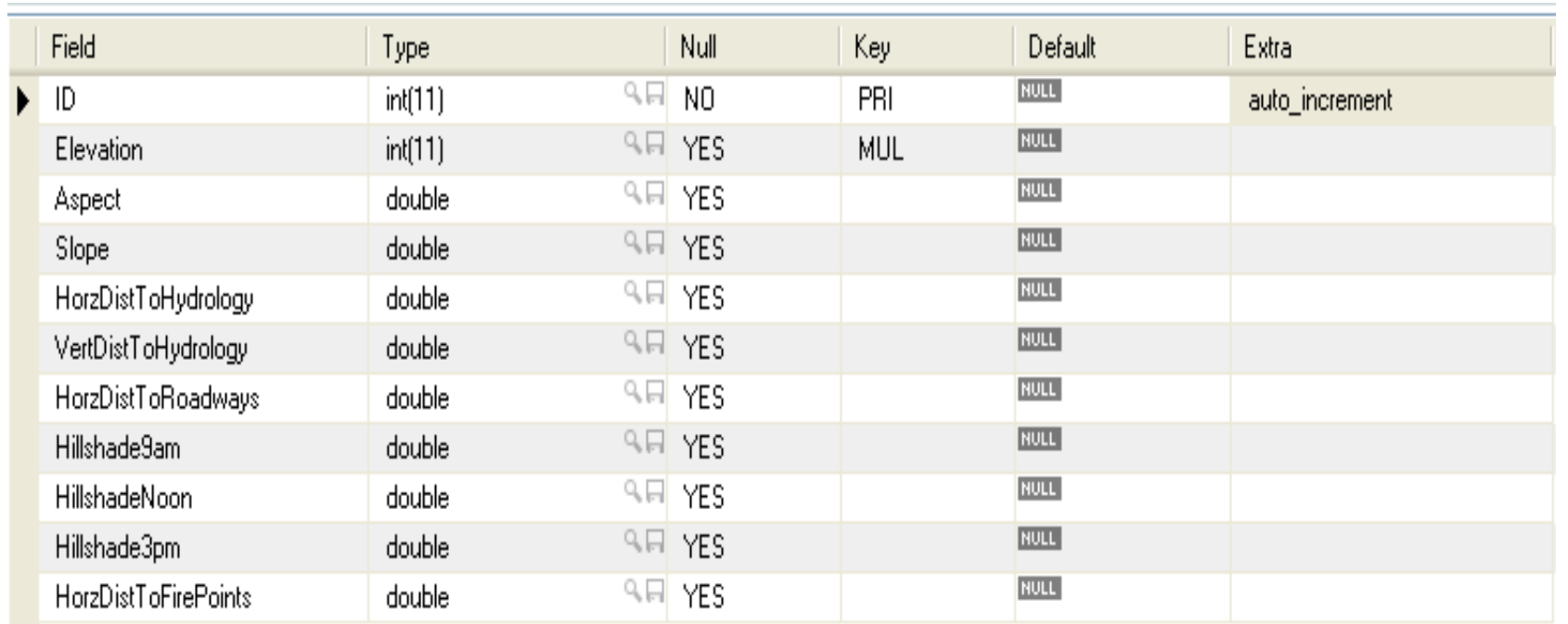

Figure 4: Forest Cover Type Dataset Schema

\begin{tabular}{|c|c|c|c|c|c|c|c|c|c|c|c|c|c|}
\hline & ID & Elevation & Aspect & Slope & HorzDist oHydt... & VertDist ohydyd... & Hord ist ToRoa... & Hillshadegam & HillshadelNoon & Hillshade3pm $H$ & HordDist of & SFilie... & \\
\hline \multirow[t]{19}{*}{1} & 1 & 2590 & 56 & 2 & 212 & 6 & 390 & 220 & 235 & 151 & & 6225 & $\wedge$ \\
\hline & 2 & 2804 & 139 & $g$ & 268 & 65 & 3180 & 234 & 238 & 135 & & 6121 & \\
\hline & 3 & 2785 & 155 & 18 & 242 & 118 & 3090 & 238 & 238 & 122 & & 6211 & \\
\hline & 4 & 2595 & 45 & 2 & 153 & .1 & 391 & 220 & 234 & 150 & & 6172 & \\
\hline & 5 & 2579 & 132 & 6 & 300 & .15 & 67 & 230 & 237 & 140 & & 6031 & \\
\hline & 6 & 2606 & 45 & 7 & 270 & 5 & 633 & 222 & 225 & 138 & & 6256 & \\
\hline & 7 & 2605 & 49 & 4 & 234 & 7 & 573 & 222 & 230 & 144 & & 6228 & \\
\hline & 8 & 2617 & 45 & 9 & 240 & 56 & 666 & 223 & 221 & 133 & & 6244 & \\
\hline & 9 & 2612 & 59 & 10 & 247 & 11 & 636 & 228 & 219 & 124 & & 6230 & \\
\hline & 10 & 2612 & 201 & 4 & 180 & 51 & 735 & 218 & 243 & 161 & & 6222 & \\
\hline & 11 & 2886 & 151 & 11 & 371 & 26 & 5253 & 234 & 240 & 136 & & 4051 & \\
\hline & 12 & 2742 & 134 & 22 & 150 & 69 & 3215 & 248 & 224 & 92 & & 6091 & \\
\hline & 13 & 2609 & 214 & 7 & 150 & 46 & 771 & 213 & 247 & 170 & & 6211 & \\
\hline & 14 & 2503 & 157 & 4 & 67 & 4 & 674 & 224 & 240 & 151 & & 5600 & \\
\hline & 15 & 2495 & 51 & 7 & 42 & 2 & 752 & 224 & 225 & 137 & & 5576 & \\
\hline & 16 & 2610 & 259 & 1 & 120 & 1 & 607 & 216 & 239 & 161 & & 6096 & \\
\hline & 17 & 2517 & 72 & 7 & 85 & 6 & 595 & 228 & 227 & 133 & & 5607 & \\
\hline & 18 & 2504 & 0 & 4 & 95 & 5 & 691 & 214 & 232 & 156 & & 5572 & \\
\hline & 10 & 2Еก & 30 & F & or & in & $7 \times 1$ & $m n$ & $n 0$ & $11 \mathrm{~A}$ & & REEF & $\underline{\underline{v}}$ \\
\hline \multicolumn{8}{|c|}{100000 wows ferched in 2.61915 (0.0299s) } & f Edit & $\checkmark$ Apply Changes & $X$ Dissard changes & || || F First & M last & $P_{\text {Search }}$ \\
\hline
\end{tabular}

Figure 5: Forest Cover Type dataset

\subsection{Experimental Results of Improved Watermarking}

Some experimental results are reported here. We run experiments on MySQL Server 5.1 using JDBC connectivity on a Windows XP Professional with a Dual Core $1.73 \mathrm{GHZ}$ Intel processor, $2 \mathrm{~GB}$ of memory, and a $10 \mathrm{~GB}$ disk drive.

\subsubsection{Imperceptibility}

The impact of watermarking on the mean and variance of values of marked attributes is reported here. This experiment was done by varying $\gamma$ from 10 to 1000 and by varying $\rho$ from 1 to 8 . We found a very negligible or no change in the mean value for all the attributes. Table 1 shows changes in variance for different attributes. The values have been rounded to the nearest integer. An empty entry indicates very little or no change. As expected, greater changes in variance occur when $\rho$ is large and $\gamma$ is small because of larger disturbances in a greater fraction of tuples. Overall, the changes are insignificant given the amount of original variance. Note that if these changes seem significant, $v, \rho$ and $\gamma$ parameters can be adjusted to reduce the impact of watermarking on the data. 
Table 1: Change in variance of different attributes after watermarking

\begin{tabular}{|c|c|c|c|c|c|c|c|c|c|c|c|}
\hline & & & \multicolumn{3}{|c|}{$\gamma=1000$} & \multicolumn{3}{|c|}{100} & \multicolumn{3}{|c|}{10} \\
\hline Attribute & Mean & Variance & $\begin{array}{c}\rho= \\
2\end{array}$ & 4 & 8 & 2 & 4 & 8 & 2 & 4 & 8 \\
\hline Elevation & 2862 & 53534.3 & - & - & -0.4 & - & - & +1.3 & - & - & +7.8 \\
\hline Aspect & 138.1 & 10770 & - & - & - & - & - & +2.7 & $0.1 \overline{7}$ & - & +16.2 \\
\hline Slope & 11.8 & 42.2 & - & - & - & - & - & - & - & - & - \\
\hline Horz-Dist-To-Hydrology & 260.5 & 4118.4 & - & - & +1.8 & - & - & +5.6 & +0.3 & +0.4 & +33.5 \\
\hline Yert-Dist-To-Hydrology & 35.2 & 1816.6 & - & - & - & - & - & - & - & - & +2.5 \\
\hline Horz-Dist-To-Roadways & 3344.3 & $\begin{array}{r}3157206 \\
7\end{array}$ & - & $0 . \overline{2}$ & -9.3 & +0.76 & +0.5 & -11.53 & +1.7 & +3.7 & -9.5 \\
\hline Hillshade-9am & 218.2 & 437.7 & - & - & - & - & - & +0.5 & - & - & +4 \\
\hline Hillshade-Noon & 225.4 & 277.3 & - & - & +0.3 & - & - & +1.7 & - & +0.13 & +20 \\
\hline Hillsha de-3pm & 139.3 & 972 & - & - & - & - & - & +0.4 & - & - & +5.6 \\
\hline Horz-Dist-To-Fire-Points & 3589.6 & $\begin{array}{r}3173452 . \\
7\end{array}$ & - & - & +0.8 & -0.8 & +3 & +2.5 & - & +3.8 & -68 \\
\hline
\end{tabular}

When an attribute value is marked, there is 0.5 probability that the value will not change. A bit with value 1 is converted to 0 with probability 0.25 and vice versa. Thus, an original value $v$ will remain $v$ with probability 0.5 and will become $v+\varepsilon$ or $v-\varepsilon$, each with probability 0.25 . Hence, if every value of an attribute is equally likely to be selected and it is as likely that the value will be incremented as decremented then the mean and variance will not be affected significantly.

\subsubsection{Robustness}

Watermarking algorithms must be developed in such a way to make it difficult for an attacker to remove a watermark from the marked database. In particular, the watermarking algorithm should make the watermarked database robust against the following types of attacks: subset deletion attack, subset addition attack, subset alteration attack, and subset selection attack.

\subsubsection{Subset Alteration Attack}

In this type of attack, the attacker alters the tuples of the database through operations such as linear transformation. The attacker hopes by doing so to remove the watermark from the database. The Table 2 indicates that the watermark will remain in the watermarked database even if $90 \%$ of the tuples of the database are altered. This is due to the fact that the proposed algorithm embeds the same watermark everywhere in the database, making this type of attack ineffective. Percent watermark detections for different subset alterations are shown in Table 2.

Table 2: Percentage of watermark detected after subset alteration attack

\begin{tabular}{|l|l|c|c|c|c|c|c|c|c|}
\hline $\begin{array}{l}\text { \% Tuples } \\
\text { Altered }\end{array}$ & \multicolumn{9}{|c|}{ Percentage of Watemark Detected } \\
\hline & \multicolumn{3}{|c|}{$\gamma=11$} & \multicolumn{3}{c|}{$\gamma=101$} & \multicolumn{3}{c|}{$\gamma=1001$} \\
\hline & $\rho=2$ & 4 & 8 & 2 & 4 & 8 & 2 & 4 & 8 \\
\hline 10 & 100 & 100 & 100 & 100 & 100 & 98 & 95 & 95 & 95 \\
\hline 20 & 100 & 100 & 100 & 100 & 100 & 97 & 94 & 90 & 94 \\
\hline 30 & 100 & 100 & 100 & 100 & 98 & 95 & 92 & 84 & 90 \\
\hline 40 & 100 & 98 & 88 & 97 & 98 & 93 & 89 & 81 & 84 \\
\hline 50 & 100 & 96 & 83 & 90 & 97 & 90 & 83 & 78 & 80 \\
\hline 60 & 100 & 82 & 80 & 73 & 91 & 83 & 81 & 72 & 75 \\
\hline 70 & 100 & 66 & 72 & 66 & 73 & 80 & 72 & 69 & 68 \\
\hline 80 & 84 & 62 & 61 & 60 & 71 & 73 & 64 & 61 & 61 \\
\hline 90 & 65 & 56 & 47 & 57 & 62 & 63 & 61 & 53 & 58 \\
\hline 100 & 53 & 50 & 41 & 52 & 55 & 59 & 59 & 42 & 57 \\
\hline
\end{tabular}

\subsubsection{Subset Selection Attack}

In this type of attack, the attacker randomly selects a subset of the original database that might still provide value for its intended purpose. The attacker hopes by doing so that the selected subset will not contain the watermark. However, since the proposed algorithm embeds the watermark in the whole database, this attack has little impact. The entries in Table 3 indicate that the watermark will remain in the watermarked database even if the attacker selects a subset as small as $10 \%$ of the original database. That is, no matter how small the subset the attacker selects, the watermark will remain in the selected subset and thus maintain the required copyright protection.

Table 3: Percentage of watermark detected after subset selection attack

\begin{tabular}{|c|c|c|c|c|c|c|c|c|c|}
\hline \multirow{3}{*}{$\begin{array}{l}\text { \% Tuples } \\
\text { Selected }\end{array}$} & \multicolumn{9}{|c|}{ Percentage of Watemark Detected } \\
\hline & \multicolumn{3}{|c|}{$\gamma=11$} & \multicolumn{3}{|c|}{$\gamma=101$} & \multicolumn{3}{|c|}{$\gamma=1001$} \\
\hline & $p=2$ & 4 & 8 & 2 & 4 & 8 & 2 & 4 & 8 \\
\hline 10 & 100 & 100 & 100 & 90 & 95 & 88 & 72 & 75 & 80 \\
\hline 20 & 100 & 100 & 100 & 100 & 98 & 93 & 80 & 82 & 81 \\
\hline 30 & 100 & 100 & 100 & 100 & 98 & 97 & 86 & 85 & 81 \\
\hline 40 & 100 & 100 & 100 & 100 & 100 & 98 & 86 & 86 & 83 \\
\hline 50 & 100 & 100 & 100 & 100 & 100 & 100 & 92 & 90 & 83 \\
\hline 60 & 100 & 100 & 100 & 100 & 100 & 100 & 96 & 90 & 90 \\
\hline 70 & 100 & 100 & 100 & 100 & 100 & 100 & 100 & 91 & 95 \\
\hline 80 & 100 & 100 & 100 & 100 & 100 & 100 & 100 & 94 & 98 \\
\hline 90 & 100 & 100 & 100 & 100 & 100 & 100 & 100 & 98 & 100 \\
\hline 100 & 100 & 100 & 100 & 100 & 100 & 100 & 100 & 100 & 100 \\
\hline
\end{tabular}

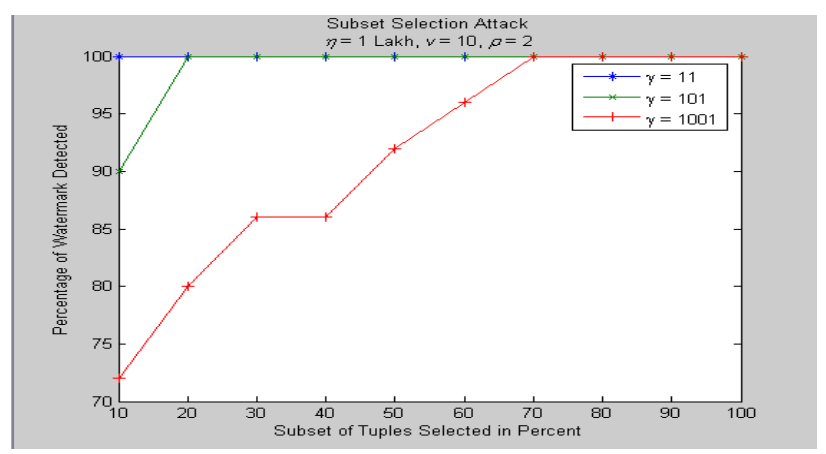

Figure 6: Subset selection attack: as the $\gamma$ value increases robustness gets decreased 
The results of percent watermark detection for selections of different subset of tuples from marked database are plotted as shown in figure 6 , and figure 7 . These plots show that as the $\gamma$ value increases the robustness of the system gets decreased.

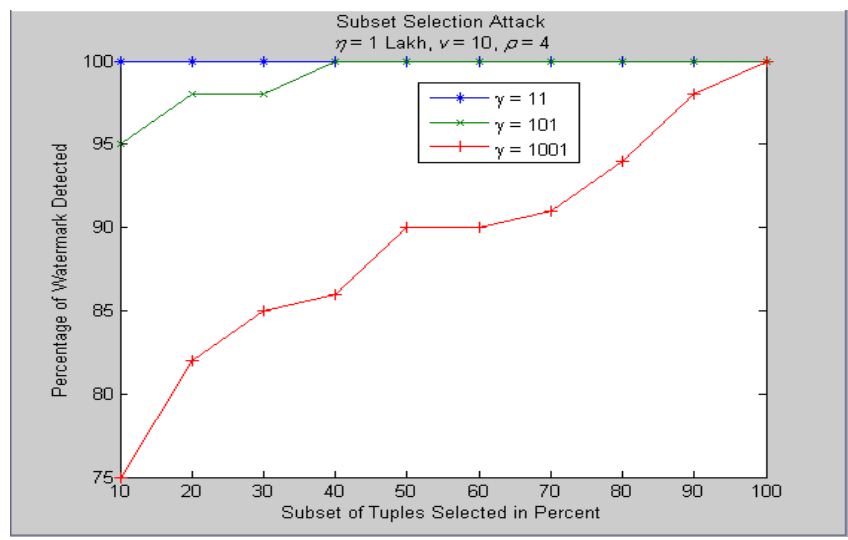

Figure 7: Subset selection attack: as the $\gamma$ value increases robustness gets decreased

\subsubsection{Subset Deletion Attack}

In this type of attack, the attacker may delete a subset of the tuples of the watermarked database hoping that the watermark will be removed. The entries in Table 4 indicate that the watermark will be removed only and only if most of the database tuples were deleted. That is, even the removal of $90 \%$ of the database will not result in removing the watermark. This is due to the fact that the proposed algorithm embeds the same watermark everywhere in the database, making this type of attack ineffective.

Table 4: Percentage of watermark detected after subset deletion attack

\begin{tabular}{|l|c|c|c|c|c|c|c|c|c|}
\hline $\begin{array}{l}\text { \% Tuples } \\
\text { Deleted }\end{array}$ & \multicolumn{7}{|c|}{ Percentage of Watemark Detected } \\
\hline \multicolumn{3}{|c|}{$\gamma=11$} & \multicolumn{3}{c|}{$\gamma=101$} & \multicolumn{3}{c|}{$\gamma=1001$} \\
\hline & \multicolumn{2}{|c|}{4} & 8 & 2 & 4 & 8 & 2 & 4 & 8 \\
\hline 0 & 100 & 100 & 100 & 100 & 100 & 100 & 100 & 100 & 100 \\
\hline 10 & 100 & 100 & 100 & 100 & 100 & 100 & 100 & 98 & 100 \\
\hline 20 & 100 & 100 & 100 & 100 & 100 & 100 & 100 & 94 & 98 \\
\hline 30 & 100 & 100 & 100 & 100 & 100 & 100 & 100 & 91 & 95 \\
\hline 40 & 100 & 100 & 100 & 100 & 100 & 100 & 96 & 90 & 90 \\
\hline 50 & 100 & 100 & 100 & 100 & 100 & 100 & 92 & 90 & 83 \\
\hline 60 & 100 & 100 & 100 & 100 & 100 & 98 & 86 & 86 & 83 \\
\hline 70 & 100 & 100 & 100 & 100 & 98 & 97 & 86 & 85 & 81 \\
\hline 80 & 100 & 100 & 100 & 100 & 98 & 93 & 80 & 82 & 81 \\
\hline 90 & 100 & 100 & 100 & 90 & 95 & 88 & 72 & 75 & 80 \\
\hline
\end{tabular}

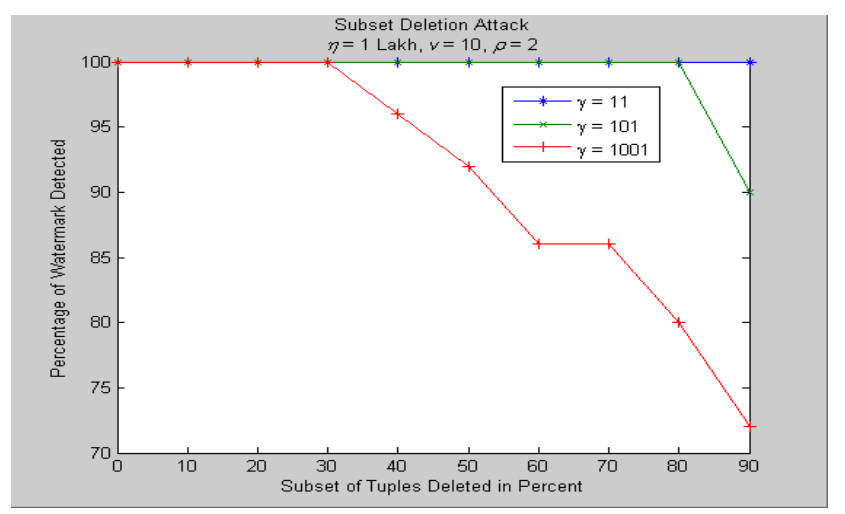

Figure 8: Subset deletion attack: as the $\gamma$ value increases robustness gets decreased
The results of percent watermark detection for deletions of different subset of tuples from marked database are plotted as shown in figure 8 , and figure 9 . These plots show that as the $\gamma$ value increases the robustness of the system gets decreased.

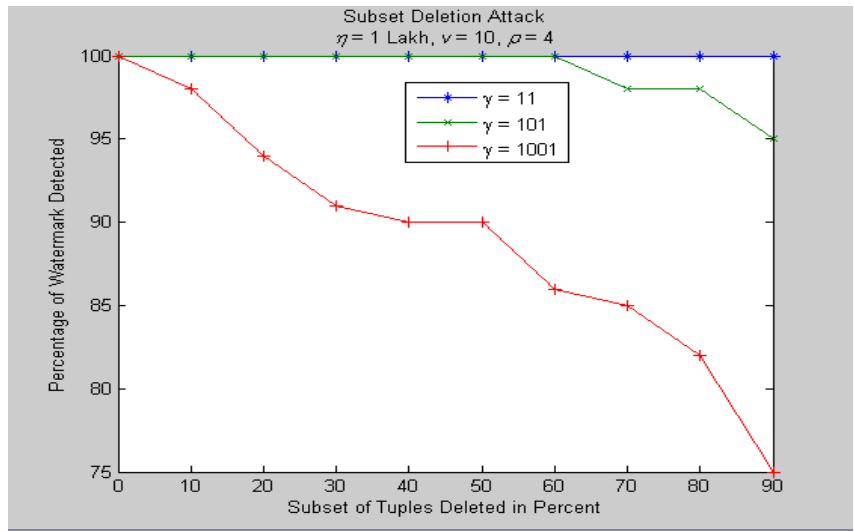

Figure 9: Subset deletion attack: as the $\gamma$ value increases robustness gets decreased

\section{CONCLUSION}

In watermarking technique we have implemented two algorithms, first is watermark insertion algorithm and second is watermark detection algorithm. Insertion algorithm first generates a bit string of fixed length and then selects a certain bit for insertion, therefore a single bit may get inserted number of times. Detection algorithm uses majority voting technique for detection of mark bits.

We have tested the watermarking algorithms for different attacks on the same real life Forest Cover Type dataset, with 1,00,000 tuples and 10 attributes available for marking. Our results show that watermark inserted by our improved watermarking technique is more robust and imperceptible than other watermarking techniques.

Our system cannot preserve the key relationships of a database and the join constraints; also the uniqueness and relative constraints of the values are not carefully considered.

Presently it marks only numeric attributes data. In the future, the proposed watermarking technique can be extended to also mark non-numeric attributes.

\section{ACKNOWLEDGMENTS}

Our thanks to all the experts who have helped us to solve the queries while developing the Improved Watermarking System. Also, special thanks to my guide Dr. Mrs. A. M. Rajurkar, for her consistent and valuable guidance during the development of the system.

\section{REFERENCES}

[1] I. J. Cox and M. L.Miller. February 1997. A review of watermarking and the importance of perceptual modeling. In Proc. of Electronic Imaging.

[2] N. F. Johnson, Z. Duric, and S. Jajodia. 2000. Information Hiding: Steganography and Watermarking Attacks and Countermeasures. Kluwer Academic Publishers.

[3] S. Katzenbeisser and F. A. Petitcolas, editors. 2000. Information Hiding Techniques for Steganography and Digital Watermarking. Artech House.

[4] Joseph J. K. 'O Ruanaidh, W. J. Dowling, and F. M. Boland. 1996. Watermarking digital images for copyright 
protection. IEEE Proceedings on Vision, Signal and Image Processing, 143(4):250-256.

[5] F. Hartung and B. Girod. 1998. Watermarking of uncompressed and compressed video. Signal Processing, 66(3):283-301.

[6] L. Boney, A. H. Tewfik, and K. N. Hamdy. June 1996. Digital watermarks for audio signals. In International Conference on Multimedia Computing and Systems, Hiroshima, Japan.

[7] S. Czerwinski. Digital music distribution and audio watermarking. Available from http://citeseer.nj.nec.com

[8] M. Atallah and S. Wagstaff. January 1999. Watermarking with quadratic residues. In Proc. of IS\&T/SPIE Conference on Security and Watermarking of Multimedia Contents.

[9] C.S.Collberg and C.Thomborson. Feb 2000. Watermarking, Tamper-Proofing, and ObfuscationTools for Software Protection. Technical Report 200003, University of Arizona.
[10] S. Benjamin, B. Schwartz, and R. Cole. 1999. Accuracy of ACARS wind and temperature observations determined by collocation. Weather and Forecasting, $14: 1032-1038$

[11] J. Kiernan, R. Agrawal, 2004. Watermarking Relational Databases, Proc. 28th Int'l Conf. Very Large Databases VLDB.

[12] Y. Li, V. Swarup, and S. Jajodia, January-March 2005. Fingerprinting Relational Databases :Schemes and specialities, IEEE transactions, Vol. 2, No. 1.

[13] J. L. Dugelay and S. Roche. 2000. A survey of current watermarking techniques. In S. Katzenbeisser and F. A. Petitcolas, editors, Information Hiding Techniques for Steganography and Digital Watermarking, chapter 6, pages 121-148. Artech House.

[14] B. Schneier. 1996. Applied Cryptography. John Wiley.

[15] A. Kerckhoffs. La cryptographie militaire. January 1983. Journal des Sciences Militaires, 9:5-38. 